\title{
Antiretroviral therapy does not reduce tuberculosis reactivation in a tuberculosis-HIV coinfection model
}

\author{
Shashank R. Ganatra, ${ }^{1}$ Allison N. Bucșan, ${ }^{2}$ Xavier Alvarez, ${ }^{1,2}$ Shyamesh Kumar, ${ }^{1}$ Ayan Chatterjee, ${ }^{1}$ Melanie Quezada, ${ }^{3,4}$ \\ Abigail Fish, ${ }^{1}$ Dhiraj K. Singh, ${ }^{1}$ Bindu Singh, ${ }^{1}$ Riti Sharan, ${ }^{1}$ Tae-Hyung Lee, ${ }^{1}$ Uma Shanmugasundaram, ${ }^{3,4}$ \\ Vijayakumar Velu, ${ }^{3,4}$ Shabaana A. Khader, ${ }^{5}$ Smriti Mehra, ${ }^{2}$ Jyothi Rengarajan, ${ }^{3,4,6}$ and Deepak Kaushal ${ }^{1}$ \\ ${ }^{1}$ Southwest National Primate Research Center (SNPRC), Texas Biomedical Research Institute, San Antonio, Texas, USA. ${ }^{2}$ Tulane National Primate Research Center (TNPRC), Covington, Louisiana, USA. \\ ${ }^{3}$ Emory Vaccine Center and ${ }^{4}$ Yerkes National Primate Research Center (YNPRC), Emory University School of Medicine, Atlanta, Georgia, USA. ${ }^{5}$ Department of Molecular Microbiology, Washington University \\ School of Medicine in St. Louis, St. Louis, Missouri, USA. 'Department of Medicine, Division of Infectious Diseases, Emory University School of Medicine, Atlanta, Georgia, USA
}

\begin{abstract}
While the advent of combination antiretroviral therapy (ART) has significantly improved survival, tuberculosis (TB) remains the leading cause of death in the HIV-infected population. We used Mycobacterium tuberculosis/simian immunodeficiency virus-coinfected (M. tuberculosis/SIV-coinfected) macaques to model M. tuberculosis/HIV coinfection and study the impact of ART on TB reactivation due to HIV infection. Although ART significantly reduced viral loads and increased CD4 ${ }^{+}$cell counts in blood and bronchoalveolar lavage (BAL) samples, it did not reduce the relative risk of SIV-induced TB reactivation in ART-treated macaques in the early phase of treatment. CD4 ${ }^{+} \mathrm{T}$ cells were poorly restored specifically in the lung interstitium, despite their significant restoration in the alveolar compartment of the lung as well as in the periphery. IDO1 induction in myeloid cells in the inducible bronchus-associated lymphoid tissue (iBALT) likely contributed to dysregulated T cell homing and impaired lung immunity. Thus, although ART was indispensable for controlling viral replication, restoring CD4 ${ }^{+} \mathrm{T}$ cells, and preventing opportunistic infection, it appeared inadequate in reversing the clinical signs of TB reactivation during the relatively short duration of ART administered in this study. This finding warrants the modeling of concurrent treatment of TB and HIV to potentially reduce the risk of reactivation of TB due to HIV to inform treatment strategies in patients with M. tuberculosis/HIV coinfection.
\end{abstract}

\section{Introduction}

The modern combination antiretroviral therapy (cART) regimen, which is known for its high efficacy and reduced toxicity, has significantly improved life expectancy in people living with HIV (PLHIV). Yet comorbidities such as tuberculosis (TB) (1) contribute significantly to all-cause mortalities in ARTadherent populations $(2,3)$, and the WHO reported $215,000 \mathrm{~TB}-$ associated deaths in HIV-infected populations in 2018. This suggests that ART fails to completely restore protective immunity to Mycobacterium tuberculosis. Despite scale-up efforts to facilitate ART accessibility to approximately $84 \%$ of notified PLHIV (4), an increased risk of mycobacterial infections with an associated risk of mortality (5) remains high within the first year of treatment in resource-limited settings $(5,6)$. Additionally, a paradoxical worsening of TB symptoms has been shown to occur soon after the initiation of ART in HIV-infected TB patients (7, 8). This is followed by a significant decline in reactivation risk

\section{Related Commentary: p. 5102}

Authorship note: JR and DK contributed equally to this work Conflict of interest: The authors have declared that no conflict of interest exists. Copyright: () 2020, American Society for Clinical Investigation. Submitted: January 16, 2020; Accepted: June 4, 2020; Published: August 24, 2020 Reference information: / Clin Invest. 2020;130(10):5171-5179. https://doi.org/10.1172/JCl136502. with increased duration of adherence to ART and associated improvements in $\mathrm{CD}^{+} \mathrm{T}$ cell counts. Although ART is associated with a significant reduction in the incidence of TB irrespective of $\mathrm{CD} 4^{+} \mathrm{T}$ cell count status (9), the lifetime risk of TB reactivation in ART-adherent cases remains 4- to 7-fold higher than in HIV-uninfected populations $(9,10) . \mathrm{CD}^{+} \mathrm{T}$ cell dysfunction and chronic immune activation are reported alongside $\mathrm{CD} 4^{+} \mathrm{T}$ cell restoration in ART-adherent PLHIV (11-13). Lung pathology was reported in $75 \%-85 \%$ of HIV/AIDS autopsies (14), and the plethora of opportunistic infections that can arise in the lung of HIV-infected individuals suggests that their lung environments remain immunologically impaired. Thus, understanding the components of TB immunity that remain impaired following HIV coinfection and ART treatment will provide insights into improving treatments for TB and HIV coinfection.

Previously, we have shown that a nonhuman primate (NHP) (rhesus macaque) model of $M$. tuberculosis/SIV coinfection effectively recapitulates many aspects of human disease, including productive SIV infection, $\mathrm{CD}^{+} \mathrm{T}$ cell depletion, $M$. tuberculosis reactivation, and chronic immune activation (15-20). Here, we extend this model to study the impact of ART on viral replication, $\mathrm{CD}^{+} \mathrm{T}$ cell restoration in various tissue compartments, chronic immune activation, and TB reactivation. Our results indicate that while ART effectively and rapidly controlled SIV replication in coinfected macaques, leading to $\mathrm{CD}^{+} \mathrm{T}$ cell restoration, it did not decrease SIV-induced TB reactivation during the period studied. 


\section{Results}

Clinical correlates of TB reactivation in ART-treated NHPs with $M$. tuberculosis/SIV coinfection. Sixteen Indian-origin mycobacteriaand SPF-4-naive rhesus macaques (Macaca mulatta) that were infected with approximately 10 CFU M. tuberculosis CDC1551 via the aerosol route $(15,21)$ converted to positive tuberculin skin tests (TSTs) after 3 weeks of M. tuberculosis exposure (Supplemental Table 1; supplemental material available online with this article; https://doi.org/10.1172/JCI136502DS1), confirming $M$. tuberculosis infection. The macaques $(n=16)$ were considered to have developed latent TB infection (LTBI), as they remained devoid of signs and symptoms of TB disease, and were enrolled for further perturbation studies (see Methods for additional details). A subset of the animals with established LTBI $(n=12)$ was challenged with $300 \mathrm{TCID}_{50}\left(50 \%\right.$ tissue culture infective dose) SIVmac $_{239}$ via the intravenous route $(15,17,22)$. Infection with SIV was confirmed by plasma viral load measurement performed at longitudinal time points, and 13 weeks after $M$. tuberculosis infection, 4 animals were initiated on the WHO-recommended ART regimen consisting of $2.5 \mathrm{mg} / \mathrm{mL}$ dolutegravir (DTG) and the 2 nucleoside reverse transcriptase inhibitors tenofovir disoproxil (PMPA) $(20 \mathrm{mg} / \mathrm{mL})$ and emtricitabine (FTC) $(30 \mathrm{mg} / \mathrm{mL})$ once daily through a single subcutaneous injection (23). The 3 experimental groups namely, LTBI, ART-naive, and ART (Figure 1A), were studied longitudinally for their clinical and pathological features and immune responses.

The ART-treated animals showed a rapid and significant $(\sim 3-\log ; P<0.00001)$ decline in plasma SIV viral loads compared with untreated controls within 2-9 weeks of ART initiation (Figure 1B). Despite the decline in SIV viral loads, however, we observed no improvement in survival, with a relative risk (RR) of TB reactivation in the ART-treated group (RR 1.23; 95\% CI 0.97-1.5) compared with ART-naive controls (Figure 1C). None of the animals in the LTBI group $(n=4)$ showed any clinical or pathological signs of TB at any point over the length of the study and were euthanized at week 24. Clinical parameters such as changes in body weight and temperature were monitored weekly (Supplemental Figure 1, A and B) and showed no significant differences between the groups. We have previously shown that elevated serum C-reactive protein (CRP) levels correlated with lung M. tuberculosis burden irrespective of SIV infection status (20). We did not detect serum CRP levels in the LTBI group, consistent with the low bacterial burdens in these animals. However, we found that CRP levels were significantly elevated $(P=0.0248)$ in both ART-naive and ART-treated groups with $M$. tuberculosis/SIV coinfection at the necropsy endpoint relative to the pre-SIV infection time point (week 9). Furthermore, we detected no differences in serum CRP levels in the ART-naive or ART-treated groups $(P=0.9073)$ at necropsy (Figure 1D). Viral loads in the acellular bronchoalveolar lavage (BAL) fluid, obtained at necropsy, were also significantly decreased $(P=$ 0.0353 ) relative to viral loads in the controls (Figure 1E). We used the RNAscope ISH assay, which detects intracellular SIV RNA targets, and performed a semiquantitative analysis of the number of cells with discrete intracellular punctate red dots. The semiquantitative scores were as follows (mean \pm SEM): $0.833 \pm 0.16$ and $0 \pm 0$ for lungs; $4 \pm 0$ and $2.4 \pm 0.4$ for spleens; and $2.83 \pm 1.16$ and $2.1 \pm$ 0.29 for lymph nodes in the ART-naive $(n=3)$ and ART-treated $(n$ =3) groups, respectively, and were not significantly different (Sup- plemental Table 2). We found that ART-naive animals displayed a high density of SIV viral RNA ${ }^{+}\left(\mathrm{VRNA}^{+}\right)$cells in lymphocyte-rich structures such as germinal centers in spleen and bronchial lymph nodes (BrLNs) and periarterial lymphatic sheaths in the spleen (Figure 1F). However, there were few $\mathrm{VRNA}^{+}$cells in the lung tissue, mostly localized in peribronchovascular bronchus-associated lymphoid tissue (BALT), and rarely in the lung parenchyma, consistent with findings from various studies reporting that lungs harbor few productively infected cells during acute SIV infection (24, 25). Besides, as early as 2 weeks after ART initiation, we detected substantially lower numbers of SIV vRNA ${ }^{+}$cells in germinal centers of the spleen and BrLNs, with minimal change in the lungs.

M. tuberculosis burden and associated lung pathology during ART therapy in M. tuberculosis/SIV coinfection. The M. tuberculosis burden was assessed in LTBI, ART-naive, and ART-treated animals by plating of BAL samples and lung, BrLN, and splenic tissues collected at necropsy. BAL samples from the LTBI group had no detectable tubercle bacilli, however, a significantly higher $M$. tuberculosis burden $(P=0.0014)$ was present in the SIV-coinfected animals irrespective of ART treatment. Similarly, SIV-coinfected animals from both ART-naive and ART-treated groups had a higher $M$. tuberculosis burden in the lungs $(P=0.0464)$, BrLNs $(P=0.0067)$, and spleen $(P=0.0402)$ compared with burdens in the LTBI group, with no significant differences between the ARTnaive and ART-treated groups $(P=0.8568, P=0.6577, P=0.2437$, respectively) (Figure 2, A-E). Although granulomas are a hallmark of TB pathology, lung granulomas are known to be heterogeneous in cellular composition, bacterial burden, and gross pathology (26). At necropsy, lungs resected from the macaques were manually dissected for macroscopic identification of granulomas and study of their gross, mycobacterial, and immune characteristics. We found a significantly higher bacterial burden $(P=0.0002)$ in granulomas from SIV-coinfected animals than in LTBI animals, with ART conferring no significant reduction $(P=0.1348)$ in granuloma $M$. tuberculosis CFU. Using the grid overlay technique described previously (27), we assessed serial lung sections for the number of lesions in each field to generate an arbitrary score corresponding to the percentage of lung involvement (Figure 2F). Animals with $M$. tuberculosis/SIV coinfection showed significantly higher numbers of TB lesions $(P=0.0240)$ compared with the LTBI-only group, whereas ART-treated animals had lesion scores comparable to those for ART-naive controls $(P=0.99)$. Gross pathology (Figure $2 \mathrm{G}$ ) showed that reactivated animals harbored numerous large granulomas, and $H \& E$ staining showed confluent granulomas with necrotic cores in both ART-naive and ART-treated animals.

Differential $C D 4^{+} T$ cell restoration in alveolar and interstitial compartments after ART therapy in M. tuberculosis/SIV coinfection of NHPs. CD4 ${ }^{+} \mathrm{T}$ cell depletion in the setting of SIV infection and its role in TB reactivation has been actively studied $(15,21)$. We assessed the effect of ART on $\mathrm{CD}^{+} \mathrm{T}$ cells in various tissues using multiparameter flow cytometry. Compared with ART-naive controls, ART-treated animals showed significantly higher $\mathrm{CD} 4^{+} \mathrm{T}$ cells in whole blood $(P=0.0427)$, BAL $(P=0.0009)$, BrLNs $(P=$ 0.0229), and spleen $(P=0.0174)$ (Figure $3, \mathrm{~A}-\mathrm{E})$, indicating that ART was able to significantly restore $\mathrm{CD} 4^{+} \mathrm{T}$ cells in these compartments. In contrast, we observed minimal restoration of $\mathrm{CD} 4^{+}$ $\mathrm{T}$ cells in the lung interstitium (supporting tissue that includes 
A

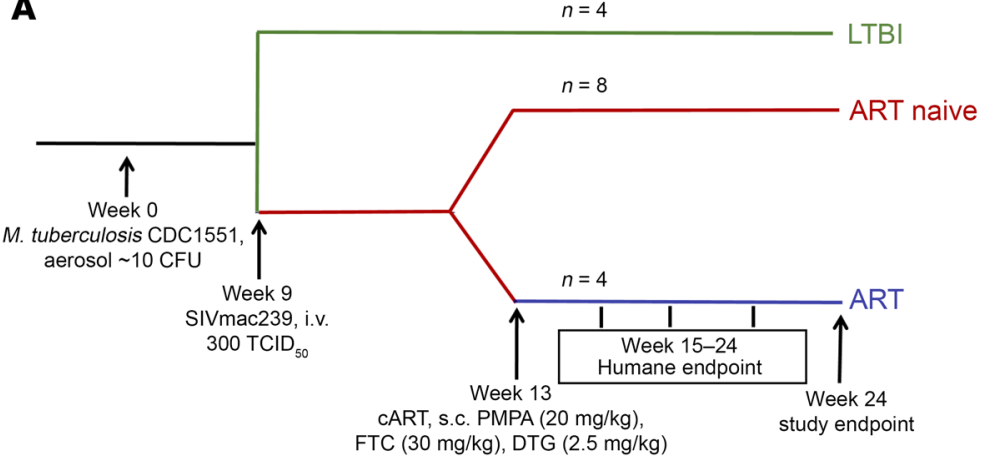

C

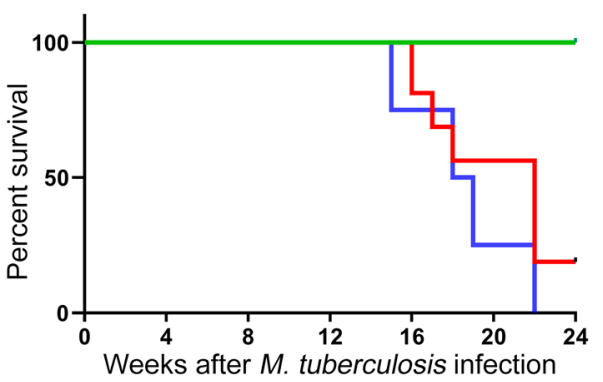

D

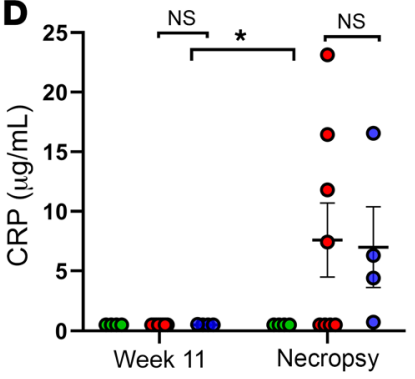

B

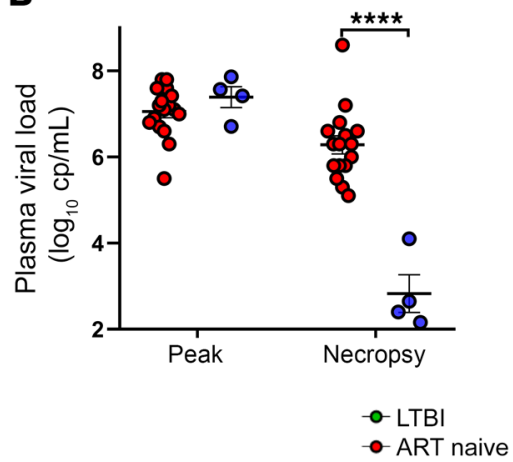

$\mathbf{F}$

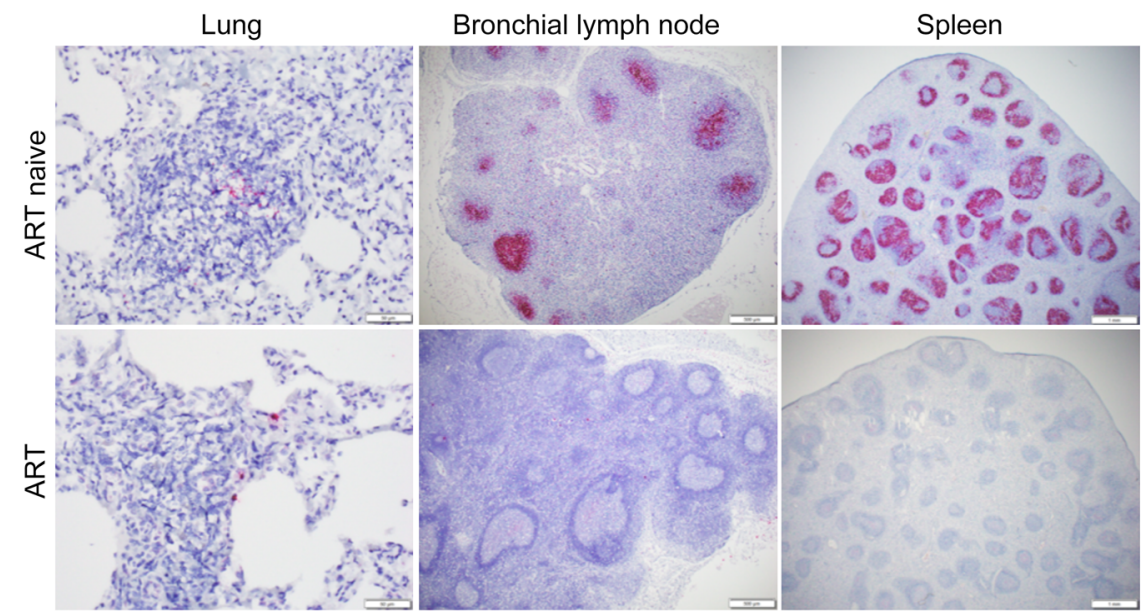

Figure 1. Clinical correlates of TB reactivation in ART-treated NHPs with M. tuberculosis/SIV coinfection. (A) Study outline. Sixteen Indian-origin rhesus macaques infected with low-dose M. tuberculosis CDC1551 via the aerosol route developed LTBI. Nine weeks after M. tuberculosis challenge, 12 these NHPs were challenged with intravenous SIVmac239. In week 13, of the $12 \mathrm{M}$. tuberculosis/SIV-coinfected NHPs, 4 received ART regimen and 8 served as ARTnaive controls. Animals with TB reactivation and signs of disease had to undergo early necropsy to meet the humane endpoints criteria set by the IACUC of TNPRC, whereas animals with no signs of active disease were necropsied in week 24. (B) Plasma viral loads were measured at the peak of SIV infection, i.e., around week 11 of infection and at the endpoint (necropsy). (C) Survival curve shows the time points (in weeks) after M. tuberculosis infection the percentage of survival. (D) Serum CRP levels were measured at 11 weeks and at the endpoint. (E) The BAL SIV RNA load was measured at necropsy. sup., supernatant. (F) An RNAscope ISH assay was performed to examine the presence of SIV RNA in tissues such as lung, BrLN, and spleen. ART treatment substantially reduced the viral particles, vRNA ${ }^{+}$cells (red), in spleen and BrLN tissue, whereas the changes were not appreciable in the sparsely infected lung parenchyma. Scale bars: $50 \mu \mathrm{m}$ (lung), $500 \mu \mathrm{m}$ (bronchial lymph node), $1 \mathrm{~mm}$ (spleen). The following 3 groups were studied: M. tuberculosis infection only, i.e., LTBI ( $n=4$, green), M. tuberculosis/SIV coinfection, i.e., ART-naive $(n=8$, red), and M. tuberculosis/SIV coinfection with ART treatment, i.e., ART $(n=4$, blue). Data represent the mean \pm SEM; error bars in the dot plots indicate the SEM. The log-rank test (Mantel-Cox) was used for comparison of survival curves in $\mathbf{C}$. ${ }^{*} P<0.05$ and ${ }^{* * *} P<0.0001$, by 2-way ANOVA with Holm-Šidák's multiple comparisons test (D) and 2-tailed Student's $t$ test (B and E). $\mathrm{cp} / \mathrm{mL}$, copies per milliliter.

alveolar epithelium and pulmonary capillary endothelium, along with perivascular and perilymphatic tissues) following ART (Figure $3, \mathrm{C}, \mathrm{F}$, and $\mathrm{G}$ ). We have previously reported a role for interstitial $\mathrm{CD} 4^{+} \mathrm{T}$ cell depletion in the dissemination of TB (28). Thus, the lack of or delay in restoration of interstitial $\mathrm{CD} 4^{+} \mathrm{T}$ cells in the lungs of ART-treated animals in our study is consistent with the reactivation of TB that occurred despite ART.

Chronic immune activation is associated with HIV, and TB/ HIV coinfection and can result in delayed functional recovery of the immune system and accelerate the progression to AIDS (29). 
A

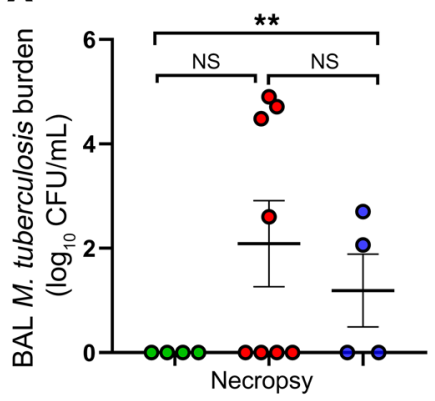

D

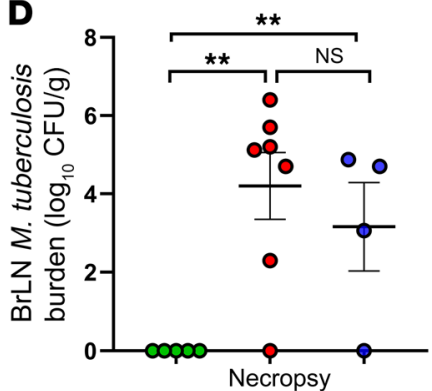

B

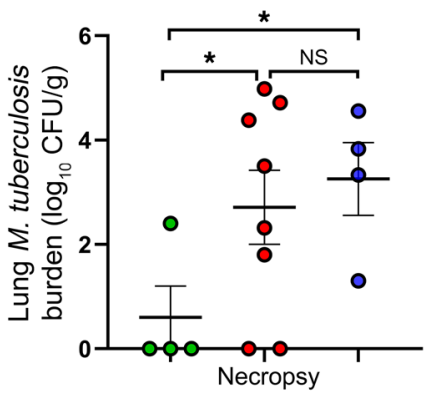

E

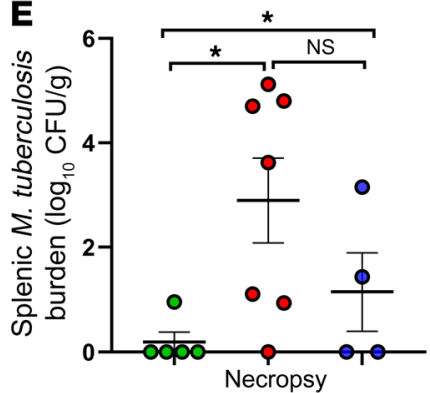

C

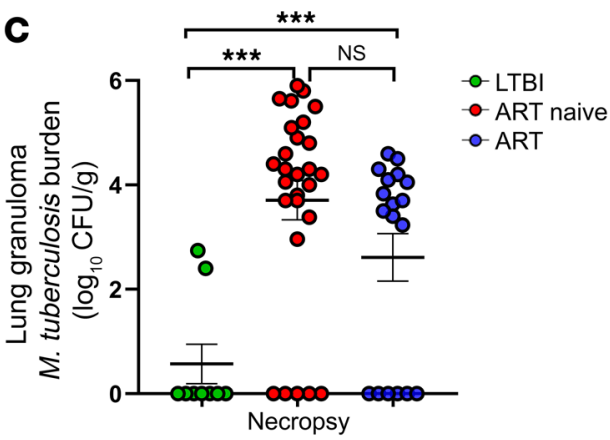

$\mathbf{F}$

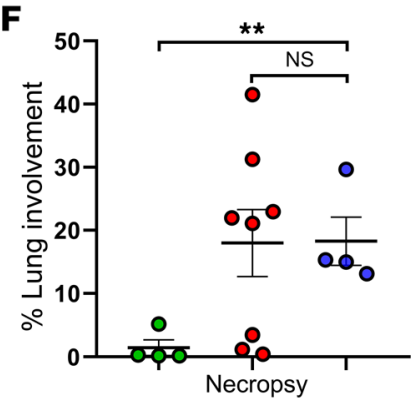

G

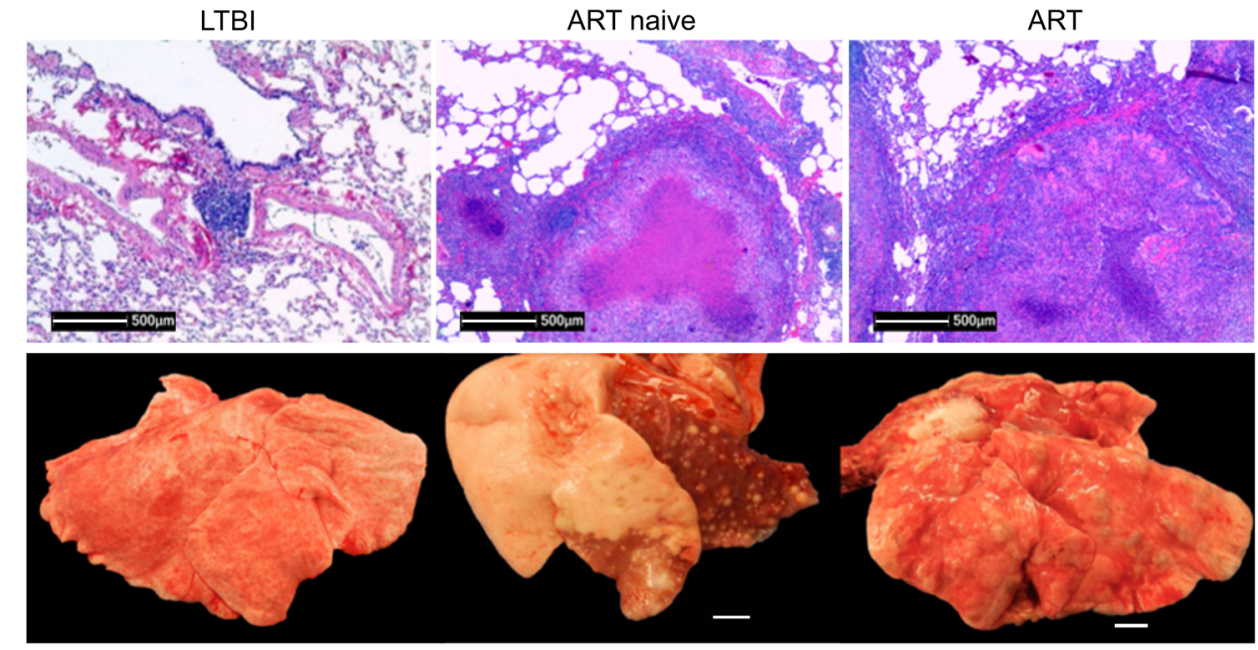

Figure 2. M. tuberculosis bacterial burden and lung pathology. M. tuberculosis bacterial burden was determined by normalizing the CFU counts to log-transformed CFU per gram of tissue. (A) M. tuberculosis CFU in the total BAL sample (cellular plus the acellular components). (B) The M. tuberculosis CFU count was normalized per gram of lung tissue collected at necropsy. (C) Multiple granulomas $(n=1-6)$ per animal were grouped according to the experimental classification of the animal; each granuloma was weighed, and its CFU count was normalized per gram of granuloma tissue. (D) BrLN M. tuberculosis burden. (E) Splenic M. tuberculosis burden. (F) The percentage of lung involvement was calculated by pathologists through extensive analysis of serially cut fresh lung samples and quantification of the number of lesions at low-power magnification. (C) Gross pathology and H\&E staining show the large granulomatous and necrotic lesions in animals with SIV-induced M. tuberculosis reactivation, whereas samples from animals with LTBI show minimal pathology. Scale bars: $500 \mu \mathrm{m}$. The following 3 groups were studied: M. tuberculosis infection only, i.e., LTBI $(n=4$, green), M. tuberculosis/SIV coinfection, i.e., ART-naive $(n=8$, red), and M. tuberculosis/SIV coinfection with ART treatment, i.e., ART ( $n=4$, blue). (A-F) Data represent the mean \pm SEM; error bars indicate the SEM. ${ }^{*} P<0.05,{ }^{*} P<0.01$, and ${ }^{* * *} P<0.001$, by 1-way ANOVA with Tukey's multiple-comparisons test.

We found no significant differences in the frequencies (Supplemental Figure 2, A-F, and Supplemental Figure 3, A and B) of HLA-DR ${ }^{+} \mathrm{CD} 4^{+} \mathrm{T}$ cells, $\mathrm{PD}-1^{+} \mathrm{CD} 4^{+} \mathrm{T}$ cells, $\mathrm{CXCR3}^{+} \mathrm{CD} 4^{+} \mathrm{T}$ cells, or $\mathrm{CCR} 6{ }^{+} \mathrm{CD} 4^{+} \mathrm{T}$ cells in blood and BAL samples from ARTnaive and ART-treated groups. However, $\mathrm{CD} 69^{+} \mathrm{CD} 4^{+} \mathrm{T}$ cell levels, a marker of early activation in response to $M$. tuberculosis antigen $(21,30)$, were higher in BAL samples from the ART-treated group compared with levels detected in the ART-naive and LTBI groups.
We noted a significant increase $(P<0.0001)$ in the frequency of $\mathrm{CXCR}^{+} \mathrm{CCR} 6^{+} \mathrm{CD} 4^{+} \mathrm{T}$ cells in ART-treated animals (Supplemental Figure 4). $\mathrm{CD}^{+} \mathrm{T}$ cells coexpressing CXCR3 and CCR6 are reported to be preferentially enriched with HIV DNA in PLHIV on highly active ART (HAART) (31). We observed a selective expansion of $\mathrm{CXCR}^{+} \mathrm{CD}^{+}$and $\mathrm{CCR} 6^{+} \mathrm{CD} 4^{+} \mathrm{T}$ cell populations within 2 weeks of cART initiation (Supplemental Figure 4, A and B). Our findings are in line with a recent report suggesting that an increase 
A

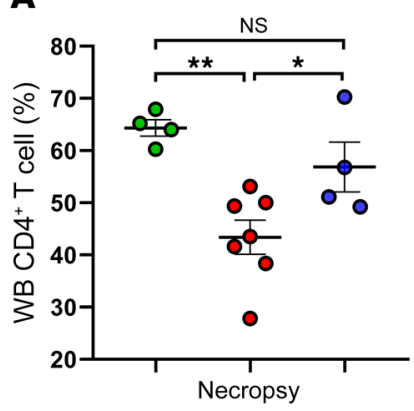

D

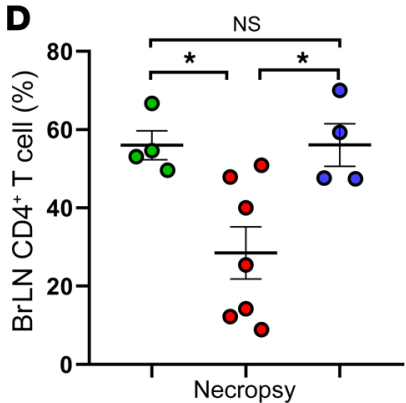

G

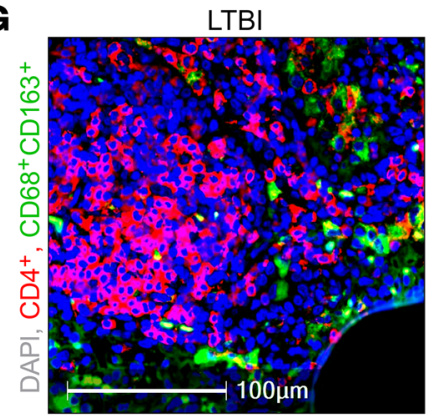

B

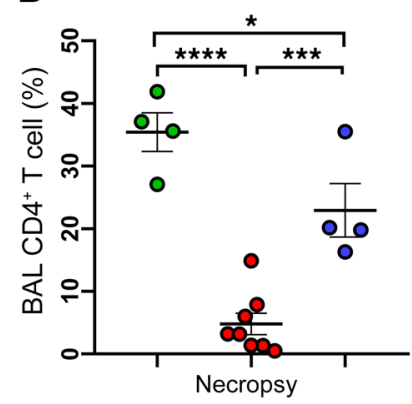

E

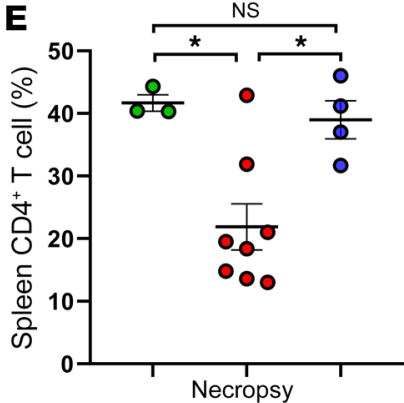

C
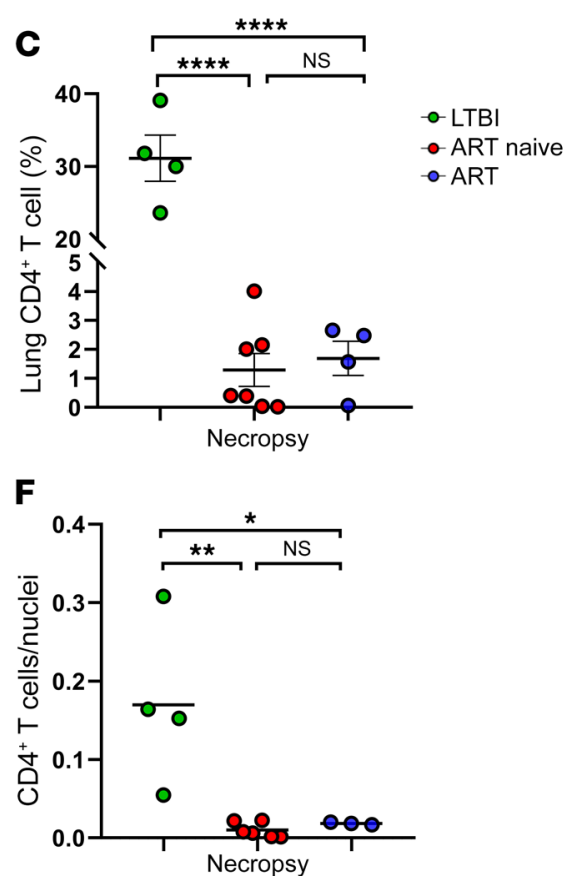
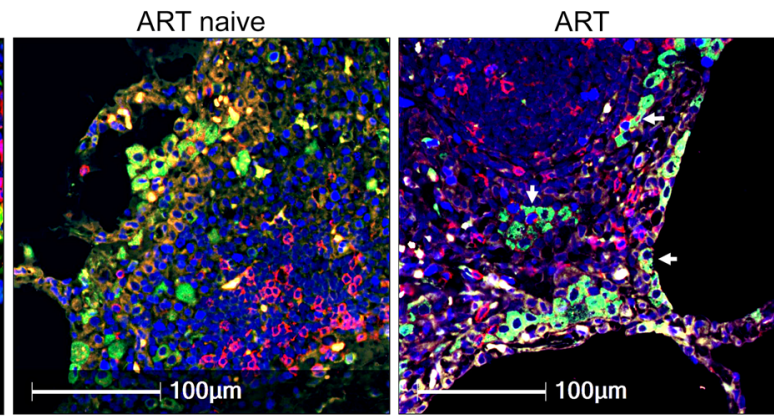

Figure 3. Differential CD4 ${ }^{+} \mathrm{T}$ cell restoration in alveolar and interstitial compartments after ART in M. tuberculosis/SIV-coinfected NHPs. Multiparameter flow cytometry was performed on single-cell suspension of various tissue samples, and whole blood was collected at necropsy from M. tuberculosis/ SIV-coinfected rhesus macaques treated with ART. The following 3 groups were studied: M. tuberculosis infection only, i.e., LTBI ( $n=4$, green), M. tuberculosis/SIV coinfection, i.e., ART-naive $(n=8$, red), and M. tuberculosis/SIV coinfection with ART, i.e., ART ( $n=4$, blue). CD4+ T cell frequency was analyzed in (A) whole blood (WB), (B) BAL, (C) lung, (D) BrLN, and (E) spleen. (F) Confocal microscopic analysis of FFPE sections from lungs harvested at the endpoint of LTBI in macaques $(n=3)$ and from ART-naive $(n=6)$ and ART-treated $(n=3)$ macaques showed CD4+ ${ }^{+}$cells (CD4+ ${ }^{+}$cells/nuclei) in lung tissue sections, counted using HALO image analysis software (Indica Labs). (C) Representative images of CD4 ${ }^{+} \mathrm{T}$ cells (red), CD68 ${ }^{+} \mathrm{CD} 163^{+}$macrophages (green), nuclei (gray), and autofluorescent RBCs (yellow). White arrowheads indicate macrophages phagocytosing CD4+ ${ }^{+}$cells in the lungs of LTBI, ART-naive, and ART groups, respectively. Scale bars: $100 \mu \mathrm{m}$. Data represent the mean $\pm \mathrm{SEM}$; error bars indicate the SEM. ${ }^{*} P<0.05,{ }^{* *} P<0.01,{ }^{* * *} P<0.001$, and ${ }^{* * * *} P<$ 0.0001 , by 1-way ANOVA with Tukey's multiple-comparisons test.

in expression of $\mathrm{CXCR} 3^{+} \mathrm{CCR} 6^{+} \mathrm{CD} 4^{+} \mathrm{T}$ cells is associated with the onset of TB-immune reconstitution inflammatory syndrome (TB-IRIS) in patients with HIV recently initiated on HAART (32).

We observed an increased presence of $\mathrm{CD} 68^{+} \mathrm{CD} 163^{+}$macrophages in lung sections from $M$. tuberculosis/SIV-coinfected animals, though the differences between ART-naive and ART-treated groups were not significant $(P=0.2654)$ (Supplemental Figure 6). This increase in myeloid cell populations could have been due to type I IFN-mediated proliferation and trafficking of M. tuberculosis-permissive innate immune cells that may further contribute to exacerbation of TB disease (33).

Dysregulation in homing of $C D 4^{+} T$ cells to $i B A L T$ in lung interstitium of $M$. tuberculosis/SIV-coinfected macaques. The presence of effector lymphoid tissue bronchus-associated lymphoid tissue
(iBALT) is known to contribute to protection against $M$. tuberculosis and prevent reactivation of latent TB (34). These iBALT structures that formed in the lungs of $M$. tuberculosis-infected macaques provide an environment for B cell maturation and antigen-specific memory effector $\mathrm{T}$ cells within the tissue (35). iBALT structures are highly organized lymphoid aggregates consisting of $B$ cell zones, serving as a germinal center, and T cell zones, harboring $\mathrm{CD}^{+}$and $\mathrm{CD} 8^{+} \mathrm{T}$ cells along with DCs and high endothelial venules $(36,37)$. $\mathrm{CD} 4^{+}$effector memory $\mathrm{T}$ cells residing in these iBALTs are targeted by SIV, resulting in their depletion and poor reconstitution despite successful control of viral replication by ART (38). We have previously demonstrated that iBALT persisted in the lungs of rhesus macaques in the setting of M. tuberculosis/ SIV coinfection, even when $\mathrm{CD} 4^{+} \mathrm{T}$ cells are depleted. Moreover, 
A

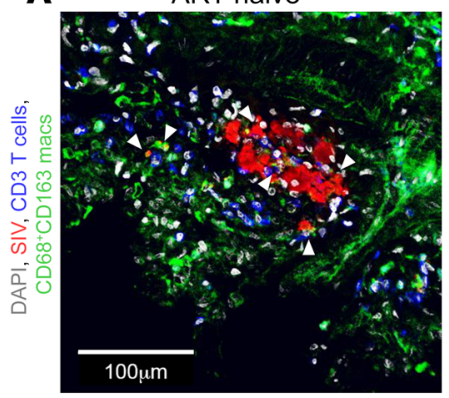

B

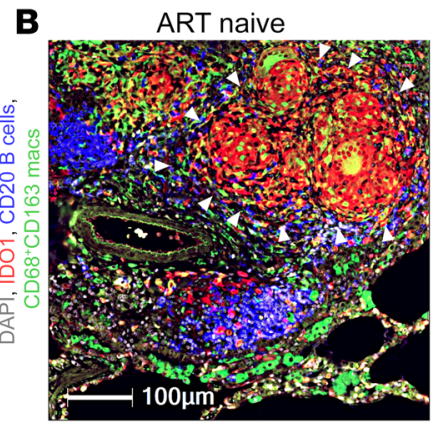

C

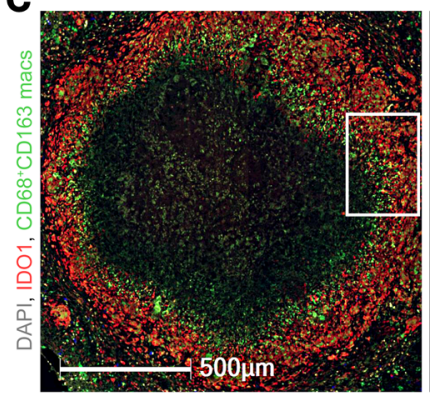

D

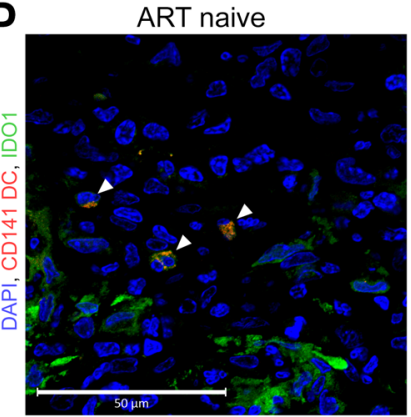

ART

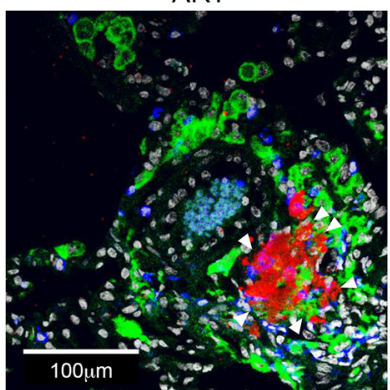

ART
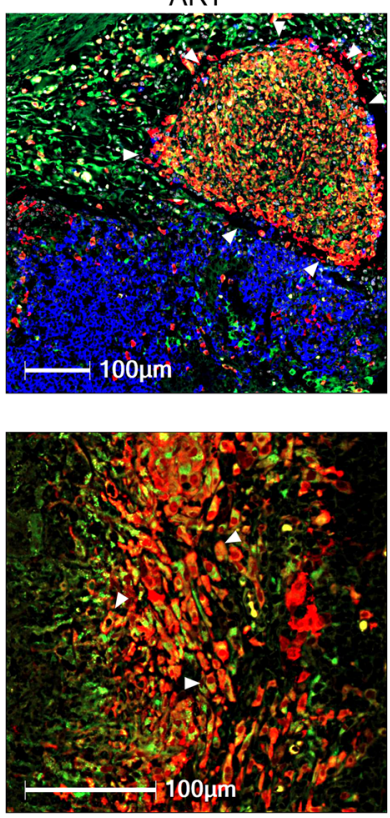

ART

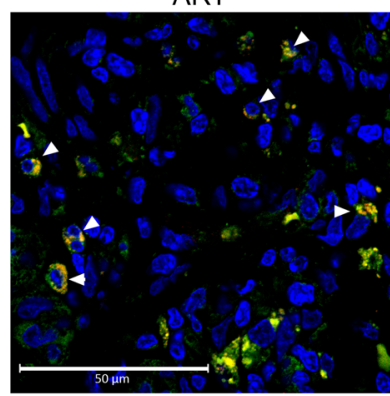

iBALT structures occupied a higher percentage of lung area in nonreactivators compared with reactivators, suggesting that the presence of iBALT correlates with protective immunity against $M$. tuberculosis reactivation in the setting of $M$. tuberculosis/SIV coinfection $(15,39)$. In this study, we examined iBALT structures in the lung using IHC. We observed a predominance of $\mathrm{CD} 68^{+} \mathrm{CD} 163^{+}$ macrophages (Figure 4A and Supplemental Figure 5, A and B) phagocytosing necrotic SIV-infected cells in the T cell zone, which is typically occupied by $\mathrm{CD}^{+} \mathrm{T}$ lymphocytes in lesions from macaques with LTBI. These productively infected macrophages and myeloid cells have been shown to serve as a reservoir for SIV in ART-suppressed macaques and are associated with high levels of immune activation (40). We have previously shown in our model of $M$. tuberculosis/SIV coinfection, through BrdU labeling,

Figure 4. Dysregulation of homing of $\mathrm{CD4}^{+} \mathrm{T}$ cells to iBALT in lung interstitium of $M$. tuberculosis/SIV-coinfected macaques. IHC staining and confocal imaging of FFPE lung sections from $M$. tuberculosis/SIV-infected macaques with or without ART. The figure is representative of 3 experimental replicates. (A) Nuclei/DAPI (gray), SIV RNA+ (red), $\mathrm{CD3}^{+}$T lymphocytes (blue), and CD68+CD163+ macrophages (Macs, green) show macrophages phagocytosing vRNA ${ }^{+}$cells present in iBALT. Scale bars: $100 \mu \mathrm{m}$. (B) Nuclei/DAPI (gray), ID01-expressing cells (red), CD20+ B lymphocytes (blue), and $\mathrm{CD}^{\circ} 8^{+} \mathrm{CD} 163^{+}$macrophages (green) identify well-organized $\mathrm{B}$ cell zones of iBALT and the presence of IDO1-expressing macrophages in the T cell zone of iBALT. Scale bars: $100 \mu \mathrm{m}$. (C) Nuclei/DAPI (gray), ID01expressing cells (red), and $C D 68^{+} C D 163^{+}$macrophages (green) of granuloma in a M. tuberculosis/SIV-coinfected animal showing that the majority of the ID01 expression is in the $\mathrm{CD68}{ }^{+} \mathrm{CD}_{163}{ }^{+}$macrophage-rich layer of granulomas in macaques with TB reactivation. Scale bars: $500 \mu \mathrm{m}$ and $100 \mu \mathrm{m}$ (enlarged inset). (D) Nuclei/DAPI (blue), ID01-expressing cells (green), and CD141+ tolerogenic DCs (red) show ID01 expression by DCs. Scale bars: 50 $\mu \mathrm{m}$. IHC staining was performed on sections of lungs from macaques with M. tuberculosis infection only, i.e., LTBI $(n=3)$, M. tuberculosis/SIV coinfection, i.e., ART-naive $(n=3)$, and M. tuberculosis/SIV coinfection with ART treatment, i.e., ART $(n=3)$ groups.

that high turnover of macrophages correlates with TB reactivation (20). Studies have shown that $\mathrm{CD}^{+} \mathrm{T}$ cell depletion after SIV infection significantly increases viral replication in macrophages and other antigen-presenting cells (41). In the face of ART, these SIV-infected macrophages serve as a reservoir by harboring latent viral genomes and contribute to viral rebound upon ART interruption (40). High turnover of macrophages contributes to macrophage persistence as antigen-presenting cells and drives chronic immune activation (42). Furthermore, a recent study also suggested that synergy between $M$. tuberculosis and SIV within lung granuloma facilitates $M$. tuberculosis bacterial dissemination and growth, thereby contributing to TB reactivation (43). Thus, our work suggests a significant dysregulation in the reconstitution of iBALT structures proximal to granulomas despite ART, and this may be one of the major mechanisms by which immune function remains impaired in $M$. tuberculosis/HIV-coinfected individuals.

Further investigation of macrophages and myeloid-derived cells in the $\mathrm{T}$ cell zone of BALT showed that these cells expressed indoleamine 2,3-dioxygenase (IDO1) (see IDO1-expressing macrophages in granulomatous lesions in Figure 4B). Our previous studies in the macaque model of $M$. tuberculosis infection showed IDO-expressing cells in the macrophage-rich layer of granulomas, which likely serves to prevent optimal interactions between $\mathrm{CD}^{+} \mathrm{T}$ cells and $M$. tuberculosis-infected antigen-presenting cells. Moreover, increased expression of IDO1 correlated with $M$. tuberculosis bacterial burden, and IDO1 expression was also associated with poorly formed iBALT. Our laboratory has previously reported that inhibition of IDO1 activity resulted in reorganization of granulomas and granuloma-associated iBALT structures, resulting in improved M. tuberculosis clearance (44). IDO1 is an immunoregulatory enzyme known to induce apoptosis of effector $\mathrm{CD} 4^{+} \mathrm{T}$ cells and promote the activation of regulatory $\mathrm{T}$ cells. Thus, expression of IDO in macrophages (Figure 4C), CD141 $1^{+}$tolerogenic DCs (Figure 4D), and other myeloid-lineage cells in ART-treated animals may promote dysregulated homing of $\mathrm{CD} 4^{+} \mathrm{T}$ cells in the $\mathrm{T}$ cell zone of iBALT and poor restoration of $\mathrm{CD} 4^{+} \mathrm{T}$ cells in the lung interstitium. Furthermore, our model can serve as a resource to 
test the efficacy of various IDO1-modulating agents in preventing $\mathrm{TB}$ reactivation and ameliorating chronic immune activation.

\section{Discussion}

Although the macaque model of $M$. tuberculosis/HIV coinfection has been extensively used, ours is the first report to our knowledge on the implementation of ART in this model system. Our results indicate that ART significantly reduces SIV viral loads in all tissue compartments. Furthermore, significant restoration of $\mathrm{CD} 4^{+} \mathrm{T}$ cell levels was observed in the periphery, the alveolar compartment, as well as in extrapulmonary tissues. We clearly show, however, that ART failed to reconstitute $\mathrm{CD} 4^{+} \mathrm{T}$ cells in lung tissue during the shorter duration of ART administered in this study. We believe this demonstrates the strength of our model system, since extensive examination of the lung tissue is virtually impossible in patients. Accordingly, ART was unable to prevent SIV-induced reactivation of LTBI in the early phase of treatment. As in humans, the M. tuberculosis/SIV coinfection/ART model in rhesus macaques recapitulated the efficacy of ART in reducing viral load and reconstituting $\mathrm{CD}^{+}{ }^{+} \mathrm{T}$ cells. A meta-analysis reported an $18 \%$ pooled incidence of TB-associated IRIS in PLHIV initiated on ART (45). Thus, although ART prevents TB in many PLHIV, a substantial minority of such individuals go on to develop TB. Our results suggest that imprecise or dysregulated restoration of $\mathrm{CD}^{+} \mathrm{T}$ cells in the lung may play an important role in this process. Our success in being able to model a more widespread failure of LTBI maintenance may be due to the use of a system in which high doses $\left(300 \mathrm{TCID}_{50}\right)$ of the virus were used for coinfection via the systemic (intravenous) route. The use of a physiologically relevant low dose and a mucosal route for SIV coinfection, along with extremely low doses of M. tuberculosis sufficient to generate LTBI in our model would, however, render our model cost-prohibitive and statistically underpowered. Although in our model of $M$. tuberculosis/SIV coinfection, we challenged LTBI macaques with SIV to study the role of SIV infection in $M$. tuberculosis-specific CD $4^{+} \mathrm{T}$ cell immunity, we understand that the sequence of infection can be reversed in areas where there is an HIV and TB syndemic. Our findings of TB reactivation with SIV challenge 9 weeks after M. tuberculosis infection are in accord with other studies, in which SIV challenge given 8-10 months (16) after M. tuberculosis infection resulted in TB reactivation. Also, our model may be of value for the study of recurrent TB in PLHIV, as it explains the cause of relapse after anti-TB treatment completion (46) and the increased susceptibility to exogenous $M$. tuberculosis reinfection after curative anti-TB therapy (47).

Our findings are consistent with available human data suggesting that initiation of ART after $M$. tuberculosis/HIV coinfection may not prevent reactivation of LTBI in all individuals $(48,49)$. It is, however, conceivable that coupling ART with either isoniazid preventive therapy (IPT) or isoniazid plus rifapentine 3-month therapy (3HP) may completely or substantially prevent TB reactivation. In this regard, it is important to note that we have developed a model for LTBI treatment in macaques using 3HP (18). Furthermore, as novel treatment regimens for LTBI with greater efficacy are developed, our model could serve as the key resource to validate them. As such, our results have the potential to inform treatment approaches for the syndemic resulting from the 2 most important infectious diseases of humanity.

\section{Methods}

Animal studies. Sixteen Indian-origin rhesus macaques (Macaca mulatta) were infected with a low dose of approximately 10 CFU M. tuberculosis CDC1551 (BEI Resources, catalog NR13649) via the aerosol route. The animals were enrolled from a specific pathogen-free colony maintained at TNPRC and were tested and found to be free from SPF-4 (simian retrovirus D, SIV, STLV-1, and herpes B virus) and TB pathogens. PCR-based molecular typing was performed to study the expression of the MHC class 1 alleles MAMU $\mathrm{A}^{*} 01, \mathrm{~B}^{*} 08, \mathrm{~B}^{*} 17$, though enrollment in the study was independent of MAMU status. All animals had a positive TST after 3 weeks of exposure, confirming infection. The animals were then examined weekly for body temperature and weight and subjected to a physical examination by a board-certified veterinary clinician. Blood examination, consisting of hematology and serum biochemistry, was performed weekly, whereas BAL assessment was performed biweekly in mice under general anesthesia. LTBI was confirmed if the animals did not show signs of reactivation in the form of fever, greater than $20 \%$ weight loss, anorexia, labored breathing, and/ or increased CRP levels up to week 8 after M. tuberculosis exposure. Nine weeks after $M$. tuberculosis infection, 12 LTBI-confirmed animals were challenged with $300 \mathrm{TCID}_{50}$ SIVmac $_{239}$ (provided by the Preston Marx Laboratory, TNPRC, Covington, Louisiana, USA) via the intravenous route, whereas 4 animals served as LTBI controls. Once infection with SIV was confirmed with plasma SIV RNA viral load, the animals were randomized to the control (ART-naive) group $(n=8)$ or the treatment (ART) group $(n=4)$. Thirteen weeks after M. tuberculosis infection, animals in the treatment group were placed on a cART regimen. All animals were euthanized by week 24 , which was a predetermined study endpoint, or at earlier time points if the animals were clinically unwell and/or showed signs of TB reactivation, as determined by veterinarians, in accordance with humane endpoints criteria.

ART drug formulation. PMPA and FTC were obtained from Gilead Sciences and DTG from ViiV Healthcare. These antiretroviral drugs were administered in a formulation of a 3-drug cocktail dissolved in the vehicle kleptose following previously published doses for each drug. Each milliliter of formulation contained the reverse transcriptase inhibitors PMPA (20 mg/mL) (50-52) and FTC (30 mg/mL) (53) and the integrase inhibitor DTG $(2.5 \mathrm{mg} / \mathrm{mL})(54,55)$.

Plasma and BAL SIV viral load. To determine the efficacy of cART, BAL samples were collected biweekly by vigorous infusion of $50 \mathrm{~mL}$ sterile PBS through an orotracheal tube and aspiration of as much of the instilled volume as possible. The procedure was performed by trained veterinarians. The aspirated fluid was mixed with $10 \%$ FBS (v/v) during the transit. BAL supernatant (acellular) was stored at $-80^{\circ} \mathrm{C}$ until analysis. Plasma and BAL SIV viral loads were determined in acellular BAL supernatant by RNA extraction and subsequent reverse transcription quantitative PCR (RT-qPCR) using a probe targeting the gag gene of SIV. Plasma and BAL SIV viral load measurements were performed at the National Institute of Allergy and Infectious Diseases (NIAID), Division of AIDS (DAIDS), Nonhuman Primate Core Virology Laboratory for AIDS Vaccine Research and Development at Duke University, Durham, North Carolina, USA (contract HHSN272201800003C). The lower limit of quantification for SIV copies in the RNA in this assay was 100 copies/sample.

ISH assay-RNAscope. Rhesus macaque lungs, BrLNs, and splenic tissues were collected during necropsy immediately following euthanasia. Serial sections were prepared from formalin-fixed, paraffin- 
embedded (FFPE) tissue blocks and used for ISH with the RNAscope 2.5 HD Reagent Kit (catalog 322350, Advanced Cell Diagnostics) exactly according to the manufacturer's instructions. A SIVmac239-specific probe (catalog 312811, Advanced Cell Diagnostics) containing $83 \mathrm{ZZ}$ pairs complementary to the transcripts coded by the viral genome regions 1251- 9420 (GenBank: D01065.1) (56), which code for multiple SIV proviral genes (gag, vif, pol, tat, env, vpx, vpr, nef, rev), was used. An identical assay using a probe for the bacterial DapB gene (catalog 320751, Advanced Cell Diagnostics), which is not expressed in mammalian tissues, was used as a negative control. Positive signals were detected by counting the number of dots per cell labeled with red dye, and images were captured using an Olympus BX46 microscope and an Olympus DP27 camera (Olympus America). A semiquantitative scoring system, based on the manufacturer's recommendation (57), was used to compare gene expression levels.

Confocal microscopy. FFPE sections from the lung, spleen, and lymph nodes harvested at the study endpoint were stained with $\mathrm{H} \& \mathrm{E}$, and fluorescence IHC and ISH were performed as described previously $(44,58,59)$.

Tissue processing and flow cytometry. High-parameter flow cytometry was performed on whole blood and BAL samples collected at weeks $3,7,11,15$, and 19, and also on lung, BrLN, spleen, and granuloma tissues harvested at specific study endpoints, as described previously $(15,18,21)$. Briefly, T cell phenotypes were studied using the following antibodies: anti-CD3 (clone SP34-2), anti-CD4 (clone L200), antiCD8 (clone RPA-T8), anti-CD69 (clone FN50), anti-CXCR3 (clone 1C6/CXCR3), anti-CCR6 (clone 11A9), anti-HLA-DR (clone L243) (all purchased from BD Biosciences), and anti-PD-1 (clone EH12.2H7, BioLegend). Flow cytometric data were analyzed with the FlowJo platform using previously described gating strategies $(15,18,21)$.

Statistics. Graphs were prepared and statistical comparisons applied using GraphPad Prism, version 8 (GraphPad Software). Various statistical comparisons were performed using a 2-tailed Student's $t$ test or 1- or 2-way ANOVA (60) with Tukey's or Holm-Šidák's multiple comparisons tests when applicable. Statistical differences between groups were considered significant when the $P$ value was 0.05 or less. Data are presented as the mean \pm SEM.

Study approval. All animals were housed in the Animal Biosafety Level III (ABSL3) at TNPRC, where they were treated according to standards recommended by the Association for Assessment and Accreditation of Laboratory Animal Care (AAALAC) International and the NIH Guide for the Care and Use of Laboratory Animals (National Academies Press, 2011). This study was approved by the IACUC of TNPRC (protocols P0247R, P0324, and P0295R).

\section{Author contributions}

SRG, ANB, SAK, SM, JR, and DK designed the studies. SRG, ANB, AC, DKS, AF, THL, BS, and XA conducted the experiments and acquired the data. SRG, RS, US, VV, SM, SAK, JR, and DK analyzed and interpreted the data and contributed to writing of the manuscript.

\section{Acknowledgments}

We thank Preston Marx for sharing a validated stock of SIVmac239. We also thank the NIAID, DAIDS, Nonhuman Primate Core Virology Laboratory for AIDS Vaccine Research and Development (contract HHSN272201800003C) for help with SIV viral load assessment. We acknowledge Gilead Sciences for providing the drugs PMPA and FTC and ViiV Healthcare for providing DTG. This work was primarily supported by NIH grants R01AI111943 and R01AI123047 (to DK and JR), with additional support from NIH grants R01AI111914, R01AI134240, R01AI138587, and U19AI111211 and institutional grants from the Office of the Director, NIH P51OD011133 (to SNPRC), P30 RR00165 and P51OD011132 (to YNPRC), and P30 AI050409 (Emory University Center for AIDS Research [CFAR]).

Address correspondence to: Deepak Kaushal, Southwest National Primate Research Center, Texas Biomedical Research Institute, 8715 West Military Drive, San Antonio, Texas 78227, USA. Phone: 210.258.9445; Email: dkaushal@txbiomed.org. Or to: Jyothi Rengarajan, Emory Vaccine Center, 954 Gatewood Road, Room 1022, Atlanta, Georgia, USA. Phone: 404.727.8174; Email: jrengar@emory.edu.

ANB's present affiliation is: Vaccine Research Center, National Institutes of Allergy and Infectious Diseases (NIAID), NIH, Bethesda, Maryland, USA.
1. Spertini F, et al. Safety of human immunisation with a live-attenuated Mycobacterium tuberculosis vaccine: a randomised, double-blind, controlled phase I trial. Lancet Respir Med. 2015;3(12):953-962.

2. Antiretroviral Therapy Cohort Collaboration. Survival of HIV-positive patients starting antiretroviral therapy between 1996 and 2013: a collaborative analysis of cohort studies. Lancet HIV. 2017;4(8):e349-e356.

3. Bor J, Herbst AJ, Newell ML, Bärnighausen T. Increases in adult life expectancy in rural South Africa: valuing the scale-up of HIV treatment. Science. 2013;339(6122):961-965.

4. Meintjes G, Brust JCM, Nuttall J, Maartens G. Management of active tuberculosis in adults with HIV. Lancet HIV. 2019; 6(7):e463-e474.

5. Etard JF, et al. Mortality and causes of death in adults receiving highly active antiretroviral therapy in Senegal: a 7-year cohort study. AIDS.
2006;20(8):1181-1189.

6. Moore D, et al. Prevalence, incidence and mortality associated with tuberculosis in HIV-infected patients initiating antiretroviral therapy in rural Uganda. AIDS. 2007;21(6):713-719.

7. Narita M, Ashkin D, Hollender ES, Pitchenik AE. Paradoxical worsening of tuberculosis following antiretroviral therapy in patients with AIDS. Am J Respir Crit Care Med. 1998;158(1):157-161.

8. Lawn SD, Bekker LG, Miller RF. Immune reconstitution disease associated with mycobacterial infections in HIV-infected individuals receiving antiretrovirals. Lancet Infect Dis. 2005;5(6):361-373.

9. Suthar AB, Bärnighausen T. Antiretroviral therapy and population mortality: Leveraging routine national data to advance policy. PLoS Med. 2017;14(12):e1002469.

10. Gupta A, Wood R, Kaplan R, Bekker LG, Lawn $\mathrm{SD}$. Tuberculosis incidence rates during 8 years of follow-up of an antiretroviral treatment cohort in South Africa: comparison with rates in the community. PLoS ONE. 2012;7(3):e34156.

11. Khaitan A, Unutmaz D. Revisiting immune exhaustion during HIV infection. Curr HIV/AIDS Rep. 2011;8(1):4-11.

12. Day CL, et al. PD-1 expression on HIVspecific $\mathrm{T}$ cells is associated with T-cell exhaustion and disease progression. Nature. 2006;443(7109):350-354.

13. Macatangay BJC, et al. T cells with high PD-1 expression are associated with lower HIV-specific immune responses despite long-term antiretroviral therapy. AIDS. 2020;34(1):15-24.

14. Lanjewar DN, Duggal R. Pulmonary pathology in patients with AIDS: an autopsy study from Mumbai. HIV Med. 2001;2(4):266-271.

15. Foreman TW, et al. CD $4^{+}$T-cell-independent mechanisms suppress reactivation of 
latent tuberculosis in a macaque model of HIV coinfection. Proc Natl Acad Sci USA. 2016;113(38):E5636-E5644.

16. Diedrich CR, et al. Reactivation of latent tuberculosis in cynomolgus macaques infected with SIV is associated with early peripheral $\mathrm{T}$ cell depletion and not virus load. PLOS ONE. 2010;5(3):e9611.

17. Mehra S, et al. Reactivation of latent tuberculosis in rhesus macaques by coinfection with simian immunodeficiency virus. JMed Primatol. 2011;40(4):233-243.

18. Foreman TW, et al. Isoniazid and rifapentine treatment eradicates persistent Mycobacterium tuberculosis in macaques. Am J Respir Crit Care Med.2020;201(4):469-477.

19. Bucşan AN, et al. Mechanisms of reactivation of latent tuberculosis infection due to SIV coinfection. JClin Invest. 2019;129(12):5254-5260.

20. Kuroda MJ, et al. High turnover of tissue macrophages contributes to tuberculosis reactivation in simian immunodeficiency virus-infected rhesus macaques. JInfect Dis. 2018;217(12):1865-1874.

21. Bucşan AN, et al. Mechanisms of reactivation of latent tuberculosis infection due to SIV coinfection. J Clin Invest. 2019;129(12):5254-5260.

22. Foreman TW, et al. Nonpathologic infection of macaques by an attenuated mycobacterial vaccine is not reactivated in the setting of HIV coinfection. Am J Pathol. 2017;187(12):2811-2820.

23. Whitney JB, et al. Rapid seeding of the viral reservoir prior to SIV viraemia in rhesus monkeys. Nature. 2014;512(7512):74-77.

24. Fuller CL, et al. Restricted SIV replication in rhesus macaque lung tissues during the acute phase of infection. Am J Pathol. 2002;161(3):969-978.

25. Li Y, et al. SIV Infection of lung macrophages. PLoS ONE. 2015;10(5):e0125500.

26. Barry CE, et al. The spectrum of latent tuberculosis: rethinking the biology and intervention strategies. Nat Rev Microbiol. 2009;7(12):845-855.

27. McBride A, Konowich J, Salgame P. Host defense and recruitment of Foxp $3^{+} \mathrm{T}$ regulatory cells to the lungs in chronic Mycobacterium tuberculosis infection requires toll-like receptor 2. PLoS Pathog. 2013;9(6):e1003397.

28. Corleis B, et al. HIV-1 and SIV infection are associated with early loss of lung interstitial $\mathrm{CD} 4^{+} \mathrm{T}$ cells and dissemination of pulmonary tuberculosis. Cell Rep. 2019;26(6):1409-1418.e5.

29. Pallikkuth S, Fischl MA, Pahwa S. Combination antiretroviral therapy with raltegravir leads to rapid immunologic reconstitution in treatmentnaive patients with chronic HIV infection. J Infect Dis. 2013;208(10):1613-1623.

30. Reiley WW, et al. ESAT-6-specific CD4 T cell responses to aerosol Mycobacterium tuberculosis infection are initiated in the mediastinal lymph nodes. Proc Natl Acad Sci USA. 2008;105(31):10961-10966.

31. Khoury G, et al. Persistence of integrated HIV DNA in $\mathrm{CXCR}^{+}{ }^{+} \mathrm{CCR} 6{ }^{+}$memory $\mathrm{CD} 4^{+} \mathrm{T}$ cells in HIV-infected individuals on antiretroviral thera- py. AIDS. 2016;30(10):1511-1520.

32. Silveira-Mattos PS, et al. Differential expression of CXCR3 and CCR6 on CD4 ${ }^{+}$T-lymphocytes with distinct memory phenotypes characterizes tuberculosis-associated immune reconstitution inflammatory syndrome. Sci Rep. 2019;9(1):1502.

33. O'Garra A, Redford PS, McNab FW, Bloom CI, Wilkinson RJ, Berry MP. The immune response in tuberculosis. Annu Rev Immunol. 2013;31:475-527.

34. Marin ND, Dunlap MD, Kaushal D, Khader SA. Friend or foe: the protective and pathologica roles of inducible bronchus-associated lymphoid tissue in pulmonary diseases. J Immunol. 2019;202(9):2519-2526.

35. Moyron-Quiroz JE, et al. Persistence and responsiveness of immunologic memory in the absence of secondary lymphoid organs. Immunity. 2006;25(4):643-654.

36. Rangel-Moreno J, Hartson L, Navarro C, Gaxiola M, Selman M, Randall TD. Inducible bronchus-associated lymphoid tissue (iBALT) in patients with pulmonary complications of rheumatoid arthritis. J Clin Invest. 2006;116(12):3183-3194.

37. Hirahara K, et al. Immune cell-epithelial/mesenchymal interaction contributing to allergic airway inflammation associated pathology. Front Immunol. 2019;10:570.

38. Picker LJ, et al. IL-15 induces CD4 effector memory $\mathrm{T}$ cell production and tissue emigration in nonhuman primates. JClin Invest. 2006;116(6):1514-1524.

39. Kaushal D, et al. Mucosal vaccination with attenuated Mycobacterium tuberculosis induces strong central memory responses and protects against tuberculosis. Nat Commun. 2015;6:8533.

40. Abreu CM, et al. Infectious virus persists in $\mathrm{CD}^{+} \mathrm{T}$ cells and macrophages in antiretroviral therapy-suppressed simian immunodeficiency virus-infected macaques. J Virol. 2019;93(15):e00065-19.

41. Ortiz AM, et al. Depletion of CD4 ${ }^{+} \mathrm{T}$ cells abrogates post-peak decline of viremia in SIV-infected rhesus macaques. JClin Invest. 2011;121(11):4433-4445.

42. Clayton KL, et al. Resistance of HIV-infected macrophages to $\mathrm{CD} 8^{+} \mathrm{T}$ lymphocyte-mediated killing drives activation of the immune system. Nat Immunol. 2018;19(5):475-486.

43. Diedrich CR. SIV Mycobacterium tuberculosis synergy within the granuloma accelerates the reactivation pattern of latent tuberculosis [preprint]. Posted on bioRxiv February 21, 2020. https://doi. org/10.1101/2020.02.21.959353.

44. Gautam US, et al. In vivo inhibition of tryptophan catabolism reorganizes the tuberculoma and augments immune-mediated control of $\mathrm{MyCO}$ bacterium tuberculosis. Proc Natl Acad Sci USA. 2018;115(1):E62-E71.

45. Namale PE, Abdullahi LH, Fine S, Kamkuemah M, Wilkinson RJ, Meintjes G. Paradoxical TB-IRIS in HIV-infected adults: a systematic review and meta-analysis. Future Microbiol.
2015;10(6):1077-1099.

46. Sterling TR, et al. Relapse rates after shortcourse (6-month) treatment of tuberculosis in HIV-infected and uninfected persons. AIDS 1999;13(14):1899-1904.

47. van Rie A, et al. Exogenous reinfection as a cause of recurrent tuberculosis after curative treatment. N Engl J Med. 1999;341(16):1174-1179.

48. Rangaka MX, et al. Isoniazid plus antiretroviral therapy to prevent tuberculosis: a randomised double-blind, placebo-controlled trial. Lancet. 2014;384(9944):682-690.

49. TEMPRANO ANRS 12136 Study Group, et al. A trial of early antiretrovirals and isoniazid preventive therapy in Africa. $N$ Engl JMed. 2015;373(9):808-822.

50. Tsai CC, et al. Prevention of SIV infection in macaques by (R)-9-(2-phosphonylmethoxypropyl)adenine. Science. 1995;270(5239):1197-1199.

51. Silvera P, et al. Effect of PMPA and PMEA on the kinetics of viral load in simian immunodeficiency virus-infected macaques. AIDS Res Hum Retroviruses. 2000;16(8):791-800.

52. Mavigner M, et al. Simian immunodeficiency virus persistence in cellular and anatomic reservoirs in antiretroviral therapy-suppressed infant rhesus macaques. J Virol. 2018;92(18):e00562-18.

53. Klatt NR, et al. CD8 $8^{+}$lymphocytes control viral replication in SIVmac239-infected rhesus macaques without decreasing the lifespan of productively infected cells. PLoS Pathog. 2010;6(1):e1000747.

54. Del Prete GQ, et al. Short communication: comparative evaluation of coformulated injectable combination antiretroviral therapy regimens in simian immunodeficiency virus-infected rhesus macaques. AIDS Res Hum Retroviruses. 2016;32(2):163-168.

55. Van Rompay KKA, et al. Dolutegravir monotherapy of simian immunodeficiency virus-infected macaques selects for several patterns of resistance mutations with variable virological outcomes. JVirol. 2019;93(2):e01189-18.

56. GenBank. D01065.1: Simian immunodeficiency virus proviral gag, vif, pol, tat, env, vpx, vpr, nef, rev genes, complete and partial cds. https://www. ncbi.nlm.nih.gov/nuccore/D01065.1. Updated July 24, 2016. Accessed July 8, 2020.

57. Acdbio. RNAscope troubleshooting guide. https://acdbio.com/technical-support/solutions. Accessed July 8, 2020.

58. Gautam US, et al. DosS Is required for the complete virulence of Mycobacterium tuberculosis in mice with classical granulomatous lesions. Am J Respir Cell Mol Biol. 2015;52(6):708-716.

59. Shanmugasundaram U, et al. Pulmonary Mycobacterium tuberculosis control associates with CXCR3-and CCR6-expressing antigen-specific Th1 and Th17 cell recruitment. JCI Insight. 2020;5(14):e137858.

60. Shcherbo D, et al. Bright far-red fluorescent protein for whole-body imaging. Nat Methods. 2007;4(9):741-746. 